\title{
The program «Moments of inertia» for calculating the moments of inertia of the rotational motion of molecules
}

\author{
(C) Mikhail N. Koverda, ${ }^{1+}$ Anna A. Koverda, ${ }^{2}$ and Eugeny N. Ofitserov ${ }^{1 *}$ \\ ${ }^{1}$ D.I. Mendeleev University of Chemical Technology of Russia. Miusskaya Sq., 9. Moscow, 125047. Russia. \\ Phone: +7 (499) 978-86-60. E-mail:m.kov@pm.me \\ ${ }^{2}$ Organic and Analitical Chemistry Division. Yaroslavl State Technical University. \\ Moscovsky Ave., 88. Yaroslavl, 150023. Russia. Phone: +7 (4852) 44-05-29.E-mail: a.koverda@pm.me
}

\section{*Supervising author; ${ }^{+}$Corresponding author}

Keywords: moment of inertia of rotational motion, computational chemistry.

\begin{abstract}
The moment of inertia of the rotational motion $I$, as a descriptor of the spatial structure of the molecule, which determines the properties of a substance, in accordance with the works of recent years, begins to acquire significance in the study of the «structure - property» dependencies, allowing one to describe the change in the properties of compounds in homologous series and address odd homologues. The problem is that there is no universal and transparent method for calculating the moments of inertia of the rotational motion of molecules. Researchers are trying to solve this problem in various ways: presenting the molecule as its carbon chain and calculating the moments of inertia only for it, neglecting the contribution of other atoms, manually calculating the moments of inertia for small simple molecules, based on their estimated geometry, extracting intermediate results from quantum chemical calculations of the program packages like Gaussian or Gamess. We have developed a program for the exact calculation of the moments of inertia, which uses the specification of the exact geometry of the molecule in three-dimensional space using Cartesian coordinates. The program is written on Perl programming language and is available under the GNU General Public License v3.0 (free software). The program uses XYZ files as input data. The principle of the program is to iteratively calculate the inertia moments for all possible positions in the space of the axis of rotation passing through the center of mass of the calculated molecule. The minimum and maximum values of the moments of inertia obtained during the calculation correspond to two perpendicular axes of rotation of the molecule ( $x$ and $z$ ). The moment of inertia with respect to the third remaining $y$ axis is calculated after finding the canonical equation of the straight axis perpendicular to the found $x$ and $z$ axes.
\end{abstract}

\section{References}

[1] E.N. Ofitserov. An attempt to quantitatively describe the relationship of properties of the compound on its chemical structure by A.M. Butlerov. Butlerov Communications. 2011. Vol.27. No.16. P.1-14. ROI: jbc-02/11-27-16-1

[2] E.N. Ofitserov, Yu.V. Vlasenko, and V.G. Uryadov. Numerical characteristics of the structure of organic molecules. Part 16. Relationship of melting points of fluoro-, chloro-, and bromoderivatives of normal structure alkanes with moments of inertia of rotational motion. Butlerov Communications. 2014. Vol.37. No.3. P.157-168. ROI: jbc-02/14-37-3-157

[3] Igor S. Borisevich, Anastasia K. Vinogradova, Viktor M. Pojidaev and Eugeny N. Ofitserov. The connection of inertia rotary moment with the chromatographic retention time in alkanes series. New retention indices. Butlerov Communications. 2019. Vol. 57. No. 1. P.15-20. DOI: 10.37952/ROI-jbc01/19-57-1-15

[4] E.N. Ofitserov, Yu.V. Vlasenko, and V.G. Uryadov. Numerical characteristic of the structure of organic molecule. Part 14. Study of interrelationship between the temperatures of phase transitions of a series of normal structure alkanes with the inertia moments of rotational motion. Butlerov Communications. 2011. Vol. 26. No.10. P.12-25. ROI: jbc-02/11-26-10-12

[5] V.G. Uryadov, Yu.V. Vlasenko and E.N. Ofitserov. The relationship of the melting point of alkanols-1 of normal structure with the ratio of electron energy to the value of the topological index to the $2 / 3$ degre. Bulletin of the Technological University. 2015. Vol.17. No.2. P.117-120. (russian)

[6] E.N. Ofitserov, Yu.V. Vlasenko, and V.G. Uryadov. Numerical characteristic of the structure of organic molecule. Part 13. Interrelation of the geometry of normal structure alkanes with the inertia moments of rotational motion. Butlerov Communications. 2011. Vol. 26. No.10. P.1-11. ROI: jbc-02/11-26-10-1 
THE PROGRAM «MOMENTS OF INERTIA» FOR CALCULATING THE MOMENTS OF INERTIA...

[7] Learning Perl, Fifth edition, by Randal L. Schwartz, Tom Phoenix, and Brian D. Foy. Copyright 2008 O’Reilly Media, Inc., 978-0-596-52010-6.

[8] Intermediate Perl, by Randal L. Schwartz, Brian D. Foy, and Tom Phoenix. Copyright 2006 O'Reilly Media, Inc., 0-596-10206-2. 